1 Universidade Estadual do Sudoeste da Bahia (Uesb) Jequié (BA), Brasil.

laimachado18@hotmail.com

2 Universidade Estadual do Sudoeste da Bahia (Uesb), Departamento de Saúde Jequié (BA), Brasil.

robertalaise@hotmail.com

3 Universidade Estadual do Sudoeste da Bahia (Uesb), Departamento de Saúde Jequié (BA), Brasil.

juli.silva.oliveira@gmail.com

\section{Direitos sexuais e reprodutivos: influências dos materiais educativos impressos no processo de educação em sexualidade}

\author{
Sexual and reproductive rights: influences of printed educational \\ materials in the process of education in sexuality
}

Laís Machado de Souza', Roberta Laíse Gomes Leite Morais², Juliana da Silva Oliveira³
RESUMO Com o objetivo de analisar a influência dos materiais educativos impressos na aprendizagem e promoção da saúde sexual e reprodutiva, foi realizada uma pesquisa qualitativa em 6 unidades de saúde no município de Jequié (BA) por meio de entrevistas com 6 enfermeiros(as) e 12 usuárias do planejamento reprodutivo, além de observação sistemática. Após a triangulação, os dados foram analisados segundo a técnica de análise de conteúdo. Evidenciou-se que, quando os materiais educativos impressos estão associados à comunicação horizontal entre profissionais e usuários(as) e metodologias mais diversificadas, respeitando as especificidades do público-alvo, é possível alcançar aprendizagem e autonomia.

PALAVRAS-CHAVE Saúde sexual e reprodutiva; Planejamento reprodutivo; Educação em saúde; Autonomia pessoal.

ABSTRACT In order to analyze the influence of printed educational material on learning and promotion of sexual and reproductive health, this qualitative study was conducted in 6 public primary healthcare facilities in the city of Jequie (BA) through interviews with 6 nurses and 12 users of reproductive planning services, and a systematic observation. After triangulation, the data were analyzed using the technique of content analysis. It was evidenced that when printed educational material is associated with horizontal communication between the professionals and the users and more diverse methodologies related to the specificities of the target audience, it is possible to achieve learning and autonomy.

KEYWORDS Sexual and reproductive health; Reproductive planning; Health education; Personal autonomy. 


\section{Introdução}

O debate sobre os direitos sexuais e direitos reprodutivos está inserido em uma das áreas de atuação prioritárias da Atenção Básica em saúde, que é a saúde sexual e reprodutiva. Esta se configura como uma área complexa, pois envolve o cuidado dos indivíduos e famílias inseridos em contextos diversos, o que significa lidar com aspectos que vão além do biológico, tais como: sociais, culturais, econômicos, emocionais e ambientais. Esse fato ressalta a importância da qualificação e postura dos profissionais de saúde, bem como de estratégias e materiais educativos que promovam conhecimentos significativos (BRASIL, 2006; 2010).

Entre os métodos utilizados nessa área, destaca-se a educação em saúde, que é um dos elementos fundamentais na qualidade da atenção prestada. No entanto, para que o processo educacional seja efetivo, é necessário significá-lo, ou seja, ele deve basear-se principalmente na seguinte premissa: “[...] ensinar não é transferir conhecimentos, conteúdos, nem formar; é ação pela qual o sujeito dá forma, estilo ou alma a um corpo indeciso e acomodado" (FREIRE, 1996, P. 23), levando o sujeito a pensar criticamente e a desenvolver autonomia. De acordo com Pelizzari et al. (2002), a teoria do pesquisador norte-americano David Paul Ausubel propõe duas condições necessárias para isso: disposição para aprender e o conteúdo a ser aprendido, o qual tem que ser lógica e psicologicamente significativo.

Ainda de acordo com Ausubel (1963), a aprendizagem significativa é o mecanismo humano, por excelência, para adquirir e armazenar a vasta quantidade de ideias e informações representadas em qualquer campo de conhecimento. Isso significa dizer que ela não se restringe ao ensino formal, abrangendo todas as áreas e ações que possuem como pressuposto a aprendizagem em função de um objetivo, tal como a educação em saúde. Estando aí inserida, pode ser eficazmente aplicada a qualquer área, tal como à saúde sexual e reprodutiva.

Quando a aprendizagem em saúde adquire significado, consolida-se então de maneira eficaz a promoção da saúde, que se refere ao "processo de capacitação da comunidade para atuar na melhoria de sua qualidade de vida e saúde, incluindo uma maior participação no controle desse processo" (BRASIL, 2006, P. 19). É nesse ponto que é percebida a íntima relação entre promoção da saúde e aprendizagem significativa. A efetividade da primeira se estabelece mediante o desenvolvimento desta última, isso porque a aprendizagem significativa leva o usuário a desenvolver sua autonomia, que é tida como "categoria central das ações de Promoção da Saúde" (HAESER; BÜCHELE; BRZOZOWSKI, 2012, P. 606).

A promoção da saúde, por sua vez, concretiza-se, principalmente, por meio do processo educativo, que deve se dar no âmbito das interações cotidianas com o usuário dos serviços. Aliado a isso, têm-se as tecnologias educacionais e os recursos educativos que visam subsidiar essas ações. O recurso do qual trata este trabalho de pesquisa é o Material Educativo Impresso (MEI), cuja utilização é prática comum no Sistema Único de Saúde (SUS) por serem recursos valiosos na construção do conhecimento transformador e para a defesa à saúde (PIMENTA; LEANDRO; SCHALL, 2006).

O MEI precisa refletir os princípios da promoção da saúde sendo uma ferramenta que, juntamente com a interação cotidiana entre profissional e usuário, deve ampliar neste o poder de questionar, refletir, decidir e agir por si mesmos (HAESER; BÜCHELE; BRZOZOWSKI, 2012). São ferramentas a serem utilizadas nas mais diversas estratégias educativas que devem ser implementadas a partir da problematização das realidades dos usuários e por meio de práticas de promoção da saúde mais criativas e horizontais, atribuindo a estas um viés menos instrumental (VILLA, 2006).

Contudo, apesar de muito utilizado como ferramenta educativa, Monteiro e Vargas (2006) ressaltam que os materiais educativos 
ainda apresentam fundamentos educacionais e pressupostos teórico-conceituais que carecem de ser explicados e aprofundados.

Com base nisso, surge a seguinte questão norteadora: como os materiais educativos impressos sobre direitos sexuais e direitos reprodutivos podem influenciar na aprendizagem significativa e na promoção à saúde da população?

A escolha do objeto de estudo surgiu a partir de algumas inquietações a respeito de como se dá a utilização dos materiais educativos em saúde sexual e reprodutiva por parte dos profissionais de saúde - em especial o enfermeiro, responsável pelo serviço de planejamento reprodutivo na Atenção Básica -, também com relação à potencialidade do material, aliado ao processo de educação em saúde desenvolvido nas unidades básicas, gerar aprendizado significativo e atuar como ferramenta de promoção da saúde. Ainda, emergiram questionamentos relacionados a como o profissional enfermeiro e o usuário avaliam a utilização e eficácia desses materiais. Tais inquietações surgiram a partir de experiências em palestras na área educacional e de saúde, em que essa temática é muito recorrente.

Em palestras, realizadas especialmente em escolas, foi possível observar a grande relevância dos recursos visuais para o aprendizado dos estudantes, em especial sobre temas ligados a sexo e reprodução. Nesse sentido, os MEI foram ferramentas muito importantes na construção do conhecimento. Em contrapartida, surgiram diversas dificuldades relacionadas ao conteúdo, à abordagem e até à utilização de figuras ilustrativas que acabavam por gerar falhas na compreensão por parte dos estudantes.

Este trabalho torna-se relevante devido à necessidade de se entender as variáveis do processo educativo em sexualidade, responsáveis pela aprendizagem e pela aquisição de autonomia por parte da população, tomando como base a utilização de materiais educativos, em especial, a relação do profissional com esses materiais. Além disso, todo o processo produtivo e distributivo dos materiais educativos impressos demanda gastos. Portanto, é imperativo conhecer a real utilidade e a importância que tem sido dada a esses materiais, assim como a sua eficácia no processo de educação em sexualidade. Não menos importante, ainda destaca-se a relevância em nível profissional e assistencial no sentido de buscar melhorias para os serviços de educação em saúde nessa área.

Ante essas considerações, este estudo tem como objetivo geral: analisar a utilização dos MEI em saúde sexual e reprodutiva e sua influência na aprendizagem significativa e promoção da saúde da população; e como objetivos específicos: identificar as estratégias de utilização desses materiais pelos profissionais da Atenção Básica e conhecer a sua relevância como ferramentas de aprendizagem e de promoção da saúde na perspectiva de profissionais e usuários.

\section{Metodologia}

No estudo, foi utilizada a metodologia qualitativa, subsidiada pelo referencial teórico pertinente ao objeto de estudo proposto. Entende-se que a abordagem qualitativa é a que mais se adequa aos objetivos pretendidos, pois visa a realização de reflexões sobre o desenvolvimento social, buscando realizar a solução de problemas de um determinado grupo por meio do estudo das relações, representações, crenças, percepções e opiniões, como produto das interpretações que os homens fazem a respeito da realidade em que vivem e do modo como constroem seus artefatos e a si mesmos (MINAYO, 2010).

A pesquisa foi desenvolvida no município de Jequié, no estado da Bahia, entre os meses de agosto e setembro de 2014. Como cenário, foram utilizadas seis Unidades de Saúde da Família (USF), selecionadas de acordo com os seguintes critérios: equipe mínima completa de acordo com os critérios preconizados pelo Ministério da Saúde; unidades localizadas na 
zona urbana; equipe com o tempo mínimo de atuação de um ano; USF porte I, ou seja, abrigando apenas uma equipe.

A amostra constituiu-se de um profissional enfermeiro(a) e duas usuárias do serviço de planejamento familiar de cada unidade, selecionados aleatoriamente, sendo dividida em 2 grupos: grupo 1, composto por 6 profissionais de saúde (enfermeiros); e grupo 2, composto por 12 usuárias cadastradas no serviço do planejamento familiar. Os participantes foram identificados(as) neste estudo pelas letras 'E' e 'U', respectivamente, e numerados de acordo com a sequência das entrevistas.

Para a coleta de dados, foi utilizada a entrevista semiestruturada, a qual é vista como uma forma privilegiada de interação social que tem por finalidade construir informações pertinentes ao objeto de pesquisa, ao expressar a reflexão do próprio sujeito sobre a realidade que vivencia, por meios de dados que retratam suas ideias, crenças, opiniões, maneira de pensar, atitudes, emoções e comportamentos (MINAYO, 2010). Ainda, foi utilizada a observação sistemática das consultas do planejamento familiar, que possibilitou fazer uma descrição precisa do objeto investigado.

A análise dos dados foi realizada por meio da análise de conteúdo, que é uma técnica capaz de descobrir as unidades de análises, objetivando estabelecer a comunicação a qual signifique alguma coisa para o objetivo analítico a ser estudado (BARDIN, 2010).

Os dados foram analisados de acordo com os três polos cronológicos descritos: 1) a pré-análise, que é a fase de organização com a realização da leitura flutuante, e, logo após, procede-se a constituição do corpus;2) exploração do material, que é considerada como uma fase longa e cansativa que consiste na codificação, decomposição ou enumeração em função de regras previamente formuladas; sendo assim, a fase de aplicação sistemática das decisões tomadas; 3 ) tratamentos dos resultados obtidos e interpretação, a inferência e a interpretação - objetivam tornar os dados válidos e significativos.
Dessa forma, as informações obtidas foram confrontadas com informações já existentes, o que permitiu realizar amplas generalizações, tornando assim a análise de conteúdo um dos mais importantes instrumentos para fins deste trabalho. Com intuito de ampliar a análise, foi feita a triangulação dos dados coletados a partir das entrevistas e observações.

O projeto está de acordo com a Resolução 466/2012 do Conselho Nacional de Saúde, e obedece aos preceitos éticos e legais e à norma de pesquisa que envolve seres humanos (BRASIL, 2012). Nesse sentido, foi submetido ao Comitê de Ética em Pesquisa da Universidade Estadual do Sudoeste da Bahia, sendo aprovado sob o parecer número 729.357.

A coleta de dados se deu mediante consentimento dos sujeitos da pesquisa ou responsáveis, estabelecidos por meio de Termo de Consentimento Livre e Esclarecido específico que informou, entre outros dados, sobre os objetivos do estudo e possíveis riscos a que estiveram expostos. Os participantes da pesquisa tiveram garantia de sigilo e anonimato, sendo a participação na pesquisa facultativa.

\section{Resultados e discussão}

Participaram da pesquisa cinco enfermeiras e um enfermeiro na faixa etária de 26 a 38 anos de idade, todos com curso de especialização, concluído ou em andamento, nas mais diversas áreas de atuação. Quatro desses profissionais relataram já ter participado de algum curso de capacitação na área de saúde sexual e reprodutiva.

Quanto às usuárias, apresentavam idade entre 16 e 46 anos. Seis destas com o Ensino Fundamental incompleto, uma com o Ensino Fundamental completo, uma com Ensino Médio incompleto e quatro com Ensino Médio completo. Dessas, sete relataram já ter participado, na condição de 
ouvintes, de palestras com a temática saúde sexual e reprodutiva na unidade de saúde ou no local de trabalho.

Os resultados obtidos mediante entrevistas e observações foram sistematizados em cinco categorias, a saber: conhecimentos prévios; disponibilidade de MEI em saúde sexual e reprodutiva; metodologias e estratégias de entrega do MEI; linguagem, abordagem comunicativa, ilustrações e conteúdos; e importância do MEI para a aprendizagem significativa e promoção da saúde dos usuários(as). A categorização e análise dos dados estão descritos a seguir.

\section{Conhecimentos prévios}

No decorrer das entrevistas e observações, foram levantadas informações que contribuíram para identificar o grau de conhecimento dos profissionais e usuários sobre saúde sexual e reprodutiva. Todos os enfermeiros(as) dispunham de conhecimentos suficientes para a boa e efetiva realização de atividades educativas na área, e apenas um relatou nunca ter realizado curso de capacitação ou especialização sobre sexo e/ou reprodução. Esses dados refletem de forma positiva sobre o processo de trabalho do enfermeiro, pois, além da formação inicial, a educação continuada merece destaque no processo de formação do profissional de saúde, em especial, para atuar com temas complexos como este.

De acordo com Moura e Silva (2005), a capacitação profissional é tida como meio para desenvolver competência técnica, e deficiências no processo de capacitação para trabalho dos profissionais de saúde podem influenciar no seu posicionamento ante os usuários no que diz respeito à garantia de seus direitos sexuais e reprodutivos (LEMOS, 2014).

Entre as usuárias do serviço de planejamento familiar, foi possível notar insegurança ao falar sobre o assunto, ainda que utilizada linguagem adequada e contextualizada. Quando questionadas a respeito da participação em cursos, palestras e capacitações, cinco responderam negativamente. As demais relataram de forma evasiva já ter participado de palestras dentro ou fora da unidade de saúde esporadicamente, como evidenciado nas unidades de análise a seguir: "Já participei, quando eu estava grávida" (U1). "Já sim. Assisti algumas palestras, aqui no posto mesmo" (U2). "Já. Lá no... no trabalho" (U3).

É possível notar, portanto, certa carência, inclusive, nas que relataram já ter participado de palestras, pois, para que produza conhecimentos significativos, o processo educativo em saúde não pode reduzir-se a ações pontuais e esporádicas. A aprendizagem significativa deve ter uma comunicação eficaz, que respeite e conduza o aluno a imaginar-se como parte integrante desse novo conhecimento através de elos, de termos familiares a ele (AUSUBeL, 1963). Portanto, as atividades pontuais, aqui mencionadas pelos informantes, raramente darão conta de atender a esses critérios.

\section{Disponibilidade de $\mathrm{MEI}$ em saúde sexual e reprodutiva}

Quatro enfermeiros(as) afirmaram disponibilizar de MEI em suas unidades. Os tipos prevalentes de materiais identificados por eles foram: banners, folders, cartilhas e álbuns seriados, como relatados nas unidades de análise a seguir: "Nós recebemos um banner, recebemos uma cartela com todos os métodos contraceptivos e recebemos algumas cartilhas falando sobre DST" (E3). "[...] a gente tem tanto material impresso, como aqueles... banners... também, alguns materiais que foram deixados por estudantes de enfermagem" (E4). "[...] tem também das doenças... tipo, um álbum seriado" (E5).

Nogueira, Modena e Schall (2009) defendem vídeos, folhetos, panfletos, cartilhas, entre outros, como valiosos instrumentos no campo da educação em saúde pelo seu caráter informativo. Contudo, ressalta-se 
que tais materiais precisam fazer sentido para o usuário, problematizando sua realidade de forma que a informação seja capaz de gerar conhecimento.

Apesar de confirmada a existência do material, houve, em algumas dessas unidades, dificuldades na obtenção de exemplares no momento de realização da pesquisa. As justificativas apresentadas por parte dos profissionais foram as recentes reformas das unidades que demandaram armazenamento dos materiais que ainda não tinham sido reorganizados. Outros, apenas não sabiam onde encontrá-los e não dispunham de tempo, na ocasião, para procurar, o que permite-se inferir que a utilização desse recurso pelos profissionais é pouca ou nenhuma. A não utilização do recurso material, por sua vez, pode influenciar negativamente no entendimento dos(as) usuários(as) em planejamento familiar, já que o recurso visual é de fundamental importância para a assimilação da informação (OSIS ET AL., 2006).

Em duas das unidades pesquisadas, foram notadas, inclusive, insegurança e inconsistência na fala dos profissionais no que se refere à disponibilidade do material, o que demonstrou falta de conhecimento da efetiva existência deste na unidade: "Não temos aqui na unidade, mas temos disponível na Secretaria de Saúde. Aliás, nós temos, nós recebemos!" (E3). Outro profissional foi taxativo em dizer que não dispunha de MEI, mas foi possível observar a existência de alguns exemplares de panfletos sobre saúde sexual e reprodutiva em uma pequena cesta no balcão da recepção. Tais inconsistências refletem o descaso com essas ferramentas educativas, negligenciando aspectos e formas de lidar com a sexualidade humana, bem como com as técnicas de comunicação envolvidas (MOURA; SILVA, 2005).

É relevante salientar como aspecto positivo o fato de um(a) dos(as) profissionais, além de utilizar os materiais disponibilizados pelo Ministério da Saúde, elaborou também materiais próprios que buscam atender às demandas e especificidades da população. Apenas nessa unidade de saúde havia algum tipo de MEI em saúde sexual e reprodutiva disposto na sala de enfermagem, local utilizado para a realização da consulta do planejamento familiar.

Entre as usuárias entrevistadas, cinco relataram nunca ter tido acesso na unidade a MEI em saúde sexual e reprodutiva, três responderam com apenas um 'sim' e quatro atribuíram o recebimento desses materiais às atividades de sala de espera e palestras realizadas, em especial, pelos estudantes de enfermagem durante o período de estágio: "Já, já tive... distribuiu pra várias pessoas que estavam aqui no posto" (U2). "Foi... as pessoas da Uesb [Universidade Estadual do Sudoeste da Bahia] mesmo que trouxe" (U4). "Assim já... já tive aqui no posto umas meninas falando sobre eles" (U11).

\section{Metodologias e estratégias de entre- ga do MEI}

Quando questionados sobre como os(as) usuários(as) têm acesso aos MEI em saúde sexual e reprodutiva na unidade de saúde, cinco enfermeiros destacaram as atividades educativas realizadas em salas de espera e também palestras, que segundo eles, são realizadas periodicamente. "[...] dá pra fazer uma sala de espera, os grupos educativos, principalmente quando a FTC [Faculdade de Tecnologia e Ciências] está aqui que dá um suporte" (E1). "Só através da... palestra educativa. [...] a gente faz, na realidade, uma educação em saúde naquele momento" (E3). "Através de palestras... quando a gente faz e também se eles tiverem" (E4). "Sempre que a gente realiza oficinas (E5). Está tendo palestras. [...] nós fazemos na escola" (E6).

O método de distribuição de MEI em palestras é muito utilizado por profissionais de saúde, mas é necessário pôr em 
discussão a real efetividade desse processo para a promoção da saúde baseada em uma aprendizagem real por parte do público-alvo.

Outras metodologias e momentos de entrega dos materiais foram relatados pelos(as) enfermeiros(as), tais como: exposição de cartazes, entrega durante as consultas de planejamento familiar e distribuição a estudantes que solicitam para trabalhos de escola. Contudo, durante as observações nas USF, não foi identificado nenhum cartaz sobre temas ligados à saúde sexual e reprodutiva em exposição, inclusive, dentro da sala de enfermagem.

A mesma situação pôde ser notada nas consultas de planejamento, nas quais não foi utilizado nenhum material educativo no decorrer do atendimento, apesar do discurso contrário de dois dos profissionais entrevistados quando disseram: "A gente só faz um planejamento familiar na sala de espera" (E4), "ou mesmo, durante a consulta também, se houver a necessidade, a gente expõe isso pra eles" (E5).

Um dos profissionais entrevistados relatou que os usuários apenas têm acesso ao material educativo no Centro de Referência em Saúde Sexual e Reprodutiva de Jequié, e por isso não dispõe de nenhuma metodologia de entrega.

$\mathrm{O}$ que se percebe nas ações educativas do planejamento familiar é que a orientação sobre métodos anticoncepcionais é o principal tópico abordado. Pierre e Clapis (2010) afirmam que aspectos relacionados à saúde sexual e reprodutiva - como informações sobre sexualidade humana, doenças sexualmente transmissíveis ou mesmo maternidade e paternidade - são negligenciados nas consultas de planejamento. $\mathrm{O}$ que se observa são discursos bem elaborados durante as entrevistas e raras ações evidenciadas na análise observacional das condutas práticas dos profissionais enfermeiros.

Entre as usuárias entrevistadas, cinco relataram nunca ter tido acesso a MEI sobre saúde sexual e reprodutiva na unidade de saúde e três disseram ter recebido material educativo durante consulta com a enfermeira da unidade. Contudo, ficou claro que essa entrega não se dá com frequência, pois os próprios profissionais relataram só fazer uso do material quando há necessidade, não ficando claros os critérios para identificação do que chamam de 'necessidade'. O discurso das usuárias em relação à entrega dos MEI pelos(as) enfermeiros(as) foram os seguintes: " $E$... eu recebi já uma cartilha aqui, através do planejamento familiar" (U2); "Às vezes ela dá, assim, algum panfletinho, às vezes, não sempre" (U3); "Foi eu passando pela médica, pela enfermeira" (U8).

Os relatos da maioria das usuárias que afirmaram ter tido acesso ao material na unidade confirmam o discurso dos(as) enfermeiros(as) em relação à utilização de palestras como principal metodologia de distribuição deste. Uma das usuárias disse: "Às vezes a mulher que dá a palestra mesmo que faz a entrega" (U2), outra confirmou a existência dessas atividades quando disse: "Aqui estava divulgando aí entregou o folhetinho pra a gente" (U4).

Notou-se, durante as entrevistas com as usuárias, que essas atividades são realizadas, muitas vezes, por estagiários de enfermagem. Uma usuária afirmou: "Foi... as pessoas da Uesb mesmo que trouxe” (U7). Outra confirmou a contribuição de estagiárias de enfermagem, dizendo: "Quando as meninas estão aqui no posto elas falam sobre eles" (U11). Os(as) profissionais queixaram-se do número de atividades as quais se tornam responsáveis por desenvolver na unidade de saúde, elencando esse fator como relevante para as poucas ações educativas: "Gostaria de fazer mais, mas infelizmente não é possível" (E3).

Evidencia-se, portanto, que existe uma falta de articulação entre a sistematização teórico-metodológica e os chamados materiais educativos usados (ou não) pelos 
enfermeiros(as), como suporte das ações pedagógicas.

\section{Linguagem, abordagem comunicativa, ilustrações e conteúdos}

Na opinião de quatro profissionais, os MEI em saúde sexual e reprodutiva possuem linguagem, ilustrações, abordagens comunicativas e conteúdos entre 'bons' a 'excelentes': "Tem uma linguagem bem simples e popular" (E3). No entanto, ao menos um desses considera a atuação do enfermeiro essencial na atividade educativa, pois, "se for entregar só papel, a maioria fica sem entender" (E2). Esses discursos têm respaldo na ideia de que a articulação entre MEI e o processo comunicativo entre profissional e usuário está dentro da concepção de educação dialógica destinada não só a comunicar conhecimentos, mas a desenvolver e modificar atitudes (FREIRE, 1987) que são essenciais à aquisição de autonomia do usuário em torno das decisões e ações sobre sua situação de saúde sexual e reprodutiva.

Dois profissionais consideram os MEI do Ministério da Saúde "muito básicos"(E1) e que "não são adequados para a demanda da comunidade" (E4). Este último profissional considera relevante levar em conta as especificidades dos(as) usuários(as) e o contexto em que se insere a população adstrita à unidade de saúde. Em função disso, relata elaborar materiais educativos próprios compatíveis com a demanda da comunidade: "Minha comunidade é de cigano, tem muita gente ainda muito nova, muita gente que não sabe ler, então é por isso que eu tive a necessidade de fazer esses meus materiais" (E4).

Esta última fala encontra respaldo no pensamento de Freire (1987) de que a educação tem que permitir o pensamento crítico do sujeito diante da sua realidade, por isso a necessidade da elaboração de materiais educativos que reflitam e problematizem a realidade dessas usuárias.
Das sete usuárias que tiveram acesso aos MEI, seis se posicionaram em relação à linguagem, ilustrações, comunicação e conteúdo destes. Três delas os consideraram "muito explicativos" (U2, U4, U6), duas reclamaram da dificuldade em entender alguns materiais quando relataram que "às vezes dá pra entender, às vezes não" (U1, U7), e uma queixou-se da visibilidade das ilustrações dos materiais dizendo que "em alguns panfletos que entrega dá pra ver mais visível e outros não" (U3).

Uma linguagem visual que contemple personagens, cenários e vivências mais próximas do público receptor pode possibilitar a oportunidade de construir novos significados, permitindo a maior compreensão dos conteúdos (SCHALL, 2005). Materiais carregados de textos e com imagens que não ilustrem o conteúdo abordado podem confundir ainda mais os(as) usuários(as).

\section{Importância do MEl para a aprendi- zagem significativa e a promoção da saúde dos(as) usuários(as)}

Todos os profissionais entrevistados reconhecem a importância dos MEI tanto como ferramentas facilitadoras do processo de aprendizagem dos usuários quanto para a promoção da saúde destes. Em se tratando da área de saúde sexual e reprodutiva, destacam serem estes materiais de extrema importância na realização do trabalho educativo, em especial, por se tratar de uma área complexa. "Fica mais fácil de você explicar pra a população; eles ficam mais cientes e acabam entendendo melhor e vendo que a coisa é mais séria do que eles imaginam" (E3).

O MEI também auxilia no processo de comunicação com os usuários, favorecendo o desenvolvimento de uma abordagem comunicativa horizontal, essencial para o empoderamento do usuário e construção de sua autonomia por meio de um processo educativo mais participativo. Um dos(as) 
enfermeiros(as) destaca que utiliza os materiais como ferramentas para estimular a curiosidade e salienta: "Quando eles têm curiosidade, eles vêm pra perguntar e aí a gente tem oportunidade pra conversar e esclarecer" (E5). Contudo, para que o material educativo estimule a curiosidade, é fundamental conhecer a realidade do público que se quer atingir, para saber com quais códigos de comunicação, de linguagem e de valores pode-se abordá-lo (NOGUEIRA; MODENA; SCHALL, 2009). Para tanto, os mesmos autores salientam a importância do envolvimento das secretarias municipais de saúde e da participação social, no sentido de contribuir na elaboração e organização de estratégias, visando atender às necessidades locais quanto ao uso do MEI.

O material educativo, quando bem elaborado e utilizado, problematiza e contextualiza a realidade dos(as) usuários(as), e assim o conteúdo passa a ter significado para eles. Os enfermeiros entrevistados acreditam que "não adianta a gente ficar só falando não" (E4) "[...] porque com os materiais eles visualizam o que a gente tá orientando (E2)" [...] "com certeza isso tem mais impacto" (E1).

Todas as usuárias entrevistadas destacaram a importância do MEI para aquisição de informações, em especial, para a prevenção de doenças sexualmente transmissíveis, evidenciando maior ênfase à importância dos aspectos cognitivos potencializados pelos materiais: "Ensinam a prevenir muitas doenças" (U5); "Você vai adquirindo informação" (U2); "Alertando a gente das doenças, das prevenções, é sempre bom, não é?" (U4).

Contudo, para que o processo educativo em saúde seja comprometido com a formação de cidadãos ativos e autônomos, é essencial a busca de uma educação libertadora, capaz de promover a emancipação psicossocial dos sujeitos (FREIRE, 1996). Além disso, o conceito de promoção da saúde refere-se a aspectos mais amplos, atribuindo maior enfoque aos processos de saúde e condições de bem-estar gerais dos indivíduos, em detrimento da simples busca por eliminação de doenças ou diminuição de suas incidências (CZERESNIA, 2009).

\section{Conclusão}

Percebe-se que as estratégias de utilização do material educativo impresso ainda sofrem muito com a influência do modelo biomédico que impregna muitas das ações de saúde até hoje. Isso é notório quando se observa que grande parte das usuárias apenas atribui a importância dos MEI em saúde sexual e reprodutiva à obtenção de informações sobre doenças sexualmente transmissíveis, ou mesmo na tecnicidade do processo distributivo desse material.

A disponibilidade e a utilização desses materiais nas unidades pesquisadas também merecem destaque pela incoerência entre o que foi relatado por parte dos profissionais e o que foi observado em suas práticas. Nesse sentido, notou-se desconhecimento em relação aos materiais disponíveis por parte de alguns, e, em geral, as metodologias de entrega relatadas não foram evidenciadas durante a observação, em especial, durante as consultas do planejamento. Isso leva a atentar, entre outras questões, para a necessidade de ressignificar o planejamento reprodutivo de forma que ele venha atender a propósitos que vão além da anticoncepção.

Embora tenha sido uma exceção entre os profissionais deste estudo, é importante destacar a produção de materiais informativos próprios como alternativa eficaz na sua potencialização como ferramentas de promoção da saúde, em virtude de possuírem linguagem e abordagem compatíveis com as especificidades do público-alvo.

Quanto à relação desses materiais com a aprendizagem significativa e a promoção da saúde, ficou evidente que, isoladamente, os 
MEI em saúde sexual e reprodutiva pouco contribuem nesse sentido. Contudo, aliados ao processo comunicativo horizontal entre profissionais e usuários(as) e metodologias mais diversificadas e criativas que respeitem as especificidades do público-alvo, é possível alcançar aprendizagem e autonomia.

\section{Referências}

AUSUBEL, D. P. The psychology of meaningful verbal learning: New York: Grune and Stratton, 1963.

BARDIN, L. Análise de Conteúdo. Lisboa: Edições 70, 2010.

BRASIL. Ministério da Saúde. Resolução n. 466, de 12 de dezembro de 2012. Aprova as diretrizes e normas regulamentadoras de pesquisas envolvendo seres humanos. Disponível em: <http://conselho.saude.gov.br/resolucoes/2012/Reso466.pdf>. Acesso em: 17 jul. 2015.

. Ministério da Saúde. Secretaria de Atenção à Saúde. Departamento de Atenção Básica. Saúde Sexual e Saúde Reprodutiva. Brasília, DF: Ministério da Saúde, 2010.

. Ministério da Saúde. Secretaria de Atenção À Saúde. Departamento de Atenção Básica. Política Nacional de Atenção Básica. Brasília, DF: Ministério da Saúde, 2006.

CZERESNIA, D. O conceito de saúde e a diferença entre prevenção e promoção. In: CZERESNIA, D.; FREITAS, C. M. (Org.). Promoção da saúde: conceitos, reflexões, tendências. 2. ed. rev. e amp. Rio de Janeiro: Fiocruz, 2009.

FREIRE, P. Ação cultural para a liberdade e outros escritos. Rio de Janeiro: Paz e Terra, 1987.

Pedagogia da autonomia: saberes necessários à prática educativa. São Paulo: Paz e Terra, 1996.

HAESER, L. M.; BÜCHELE, F.; BRZOZOWSKI, F. S Considerações sobre a autonomia e a promoção à saúde. Physis: Revista de Saúde Coletiva, Rio de Janeiro, v. 22, n. 2, p. 605-620, 2012.
LEMOS, A. Direitos sexuais e reprodutivos: percepção dos profissionais da atenção primária em saúde. Saúde em Debate, Rio de Janeiro, v. 38, n. 101, p. 244-253, 2014

MINAYO, M. C. S. (Org.). Pesquisa Social: teoria, método e criatividade. 29. ed. Petrópolis: Vozes, 2010.

MONTEIRO, S.; VARGAS, E. (Org.). Educação, comunicação e tecnologia educacional: interfaces com o campo da saúde. Rio de Janeiro: Fiocruz, 2006.

MOURA, E. R. F.; SILVA, R. M. Competência profissional e assistência em anticoncepção. Rev. Saúde Pública, v. 39 , n. 5 , p. $795-801,2005$.

NOGUEIRA, M. J.; MODENA, C. M.; SCHALL, V. T. Materiais educativos impressos sobre saúde sexual e reprodutiva utilizados na atenção básica em Belo Horizonte, MG: caracterização e algumas considerações. RECIIS- R. Eletr. de Com. Inf. Inov. Saúde, Rio de Janeiro, v. 3, n. 4, p. 169-179, 2009.

OSIS, M. J. D. et al. Atenção ao planejamento familiar no Brasil hoje: reflexões sobre os resultados de uma pesquisa. Cad. Saúde Pública, Rio de Janeiro, v. 22, n. 11, p. 2481-2490, 2006.

PELIZZARI, A. et al. Teoria da Aprendizagem Significativa Segundo Ausubel. Rev. PEC, Curitiba, v. 2, n. 1, p. 37-42, 2002.

PIERRE, L. A. S.; CLAPIS, M. J. Planejamento familiar em Unidades de Saúde da Família. Rev. Latino-Am. Enfermagem, Ribeirão Preto, v. 18, n. 6, p. 1161-1168, 2010 .

PIMENTA, D. N.; LEANDRO, A. M. S.; SCHALL, V. T. Experiências de desenvolvimento e avaliação de 
materiais educativos sobre saúde: abordagens sócio-históricas e contribuições da antropologia visual. In: MONTEIRO, S.; VARGAS, E. (Org.). Educação, Comunicação e Tecnologia Educacional: interfaces com o campo da saúde. Rio de Janeiro: Fiocruz, 2006.

SCHALL, V. T. Histórias, jogos e brincadeiras: alternativas lúdicas para crianças e adolescentes sobre saúde e ambiente. In: MASSARANI, L. O pequeno cientista amador. Rio de Janeiro: Fiocruz, 2005.
VILLA, E. A prática educativa no cotidiano do trabalho do profissional. In: GAZZINELI, M. F.; REIS, D. C.;

MARQUES, R. (Org.). Educação em saúde: teoria, método e imaginação. Belo Horizonte: UFMG, 2006.

Recebido para publicação em dezembro de 2014

Versão final em abril de 2015

Conflito de interesses: inexistente

Suporte financeiro: não houve 